\title{
TRANSFORMATIONS OF MONA LISA: THE CASE OF A DISTANCE EDUCATION ART-AND- TECHNOLOGY PROJECT
}

\author{
Souliotou AZ* \\ University of Thessaly, Hellenic Open University, \\ University of Western Macedonia, International Hellenic University, Greece
}

\begin{abstract}
Mona Lisa by Leonardo Da Vinci has been subject to numerous and various transformations in the form of (re)interpretations, reproductions, replicas, appropriations and parodies. Mona Lisa is far more than a mere Renaissance portrait or a symbol of its time. Instead Mona Lisa is radically connected with artistic movements and practices throughout the history of art as well as with the 20th and 21st century visual culture, visual commerce and social media imagery. This paper presents an activity in a higher education Department of Early Childhood where students experimented with digital tools and made a collective artwork of digital transformations of Mona Lisa. This digital experiment was a distance education project which took place during the COVID-19 pandemic restrictions in Greece. At first, students were given examples of appropriations and parodies of Mona Lisa from the history of art as well as from the visual culture. Then students gave their own "responses" through making digitally transformed versions of Mona Lisa which they put together in a collective digital mosaic. Clones, distortions, semi-transparencies, repositions and other transformations within 75 Mona Lisa versions render this collective artwork a composition with reference to pixel structure. Students' collective artwork contributed to the deeper understanding of Da Vinci's masterpiece and increased their confidence and familiarity with Renaissance painting. The case of this activity proves that digital culture is a catalyst for art history learning and creativity in the classroom. Furthermore, this activity fosters collaborative learning through distance education and turns out to be a vehicle for empowering learners in a digital world, as well as for developing linguistic, numerical and multisensory skills through digital creativity.
\end{abstract}

Keywords: Mona Lisa, Leonardo Da Vinci, distance education, higher education, digital art, participatory practices, community resilience

\section{Introduction}

Mona Lisa by Leonardo Da Vinci has been subject to numerous and various transformations in the form of (re)interpretations, reproductions, replicas, appropriations and parodies. Originally Mona Lisa is a portrait painted with oil colours on wood (poplar panel) by Leonardo di ser Piero Da Vinci between 1503 and 1519. The sitter is probably Lisa Gherardini, also known as La Gioconda because she was the wife of the Florentine commissioner Francesco del Giocondo (Scailliérez, 2021). However, Mona Lisa is far more than a mere Higher Renaissance portrait (Augustyn, 2021) or a symbol of its time. Instead, Mona Lisa is radically connected with artistic movements and practices throughout the history of art as well as with the 20th and 21st century visual culture, visual commerce and social media imagery. 
This paper presents Collective Mona Lisa project in a higher education Department of Early Childhood where students experimented with digital tools and made a collective artwork of digital transformations of Mona Lisa. This digital experiment was a distance education project which took place during the COVID-19 pandemic restrictions in Greece in the frame of a module on art and technology. The initial idea of this project was developed by the author/instructor. The necessity for students to collaborate in distance and to improve their digital image processing skills, especially by learning how to use transform tools, led to the implementation of Collective Mona Lisa project.

While other reproductions and appropriations are composed of a single Mona Lisa which is altered or transformed by a single artist or creator, Collective Mona Lisa project presents collective mosaics with multiple Mona Lisa portraits in various transformations made by the author/instructor and the participation of 22 students. Collective Mona Lisa multiplicities with the put-togetherness of various digital transformations endow the collective piece with playfulness and simultaneity of different views, perspectives and distortions of the original.

\section{Copying and Appropriating Mona Lisa}

This section presents replicas, parodies and (re)interpretations of Mona Lisa throughout the history of art. The following artworks were showed to students of the Department of Early Childhood Education in Greece. A visual analysis of each artwork was made using the see-think-wonder technique (Ritchhart et al, 2011, Wilhelm et al, 2014) with observation, description, formal analysis and interpretation (Sayre, 2006, p.6-14) as well as deeper analysis of form, content and context -historic, socio-political and artistic- of each artwork (Belton, 1996).

The earliest replicas of Mona Lisa, which were presented to the students, were the Isleworth Mona Lisa and the Prado Mona Lisa. Students compared and contrasted the two replicas with the original using formal visual analysis. The discussion extended to art's relation with science through the comparison of the infrared reflectograms of original Mona Lisa and Prado Mona Lisa. (Louvre, 2021) Infrared reflectography reveals details hidden under the painting layers (Louvre, 2021) which enabled a detailed comparison with clear observation of the differences between the two portraits.

Then, other artworks were presented, which are inscribed in appropriation/adaptation (TATE, 2020) art practice with the (re)use of Mona Lisa, starting from Eugène Bataille's black-and-white version. Sapeck (Eugène Bataille) made the illustration The Laugh or Mona Lisa smoking a pipe for the exhibition Les Incoherents in Paris and published it in Le Rire magazine by Ernest Coquelin, known as Coquelin cadet (Cadet, 1887, p.5). This ironic illustration is associated with fumism (in French 'fumisme'), a lifestyle of artists and writers in Montmartre which constituted a form of skepticism and humor or even an art of the morbid and the bizarre where nothing (nor even Da Vinci's Mona Lisa) was sacred (Musée de Montmartre, 2021).

L.H.O.O.Q. by Marcel Duchamp was already known to students and designates a radical appropriation that puts gender, values and symbols into question. The historic and sociopolitical context of this artwork, which was made after the 1st World War, was underlined by critical resistance and nihilistic approach towards Western culture clearly manifested through the Dada movement. L.H.O.O.Q. provoked an "explosive visual shock" and was even a negation of itself (Craven, 2017, p.75). Picabia's publication of Duchamp's L.H.O.O.Q. in 391 magazine includes DADA manifest underneath 
the mustached Mona Lisa which connects DADA with nothingness. (Picabia, 1920) It is hereby interesting to note that it is mainly thanks to Duchamp's legacy that it has become possible to include any object in art, "with or without transformation" (Centre Pompidou, Direction de l'action éducative et des publics, 2005) and this was made clear to students.

Philippe Halsman's Mona Dali: What Dali sees when he looks at Mona Lisa photomontage portrait of 1954 with Dali's characteristic mustache and look inside Mona Lisa's face was also appealing to students. The title of this photomontage evokes - besides Dali's reputation as 'Avidos Dollar'- the role of Mona Lisa's viewer (Steiner, 1987: p.174). Mona/Leo (1987) by Lilian F. Schwartz is a computergenerated collage which composes of two sides: on the right side there is Da Vinci's half autoportrait and on the left side Mona Lisa's half portrait. The doubleness in the case of Mona/Leo is clear and explicit following Duchamp's tradition of gender mystery in L.H.O.O.Q. (LaFarge, 1996, p. 380). Jean-Pierre Yvaral's Composite Mona Lisa (1989) brings the portrait to digital existence in a pixilated and numerical form which makes every part of Mona Lisa calculable (Popper, 1993). Furthermore, Yvaral's Mona Lisa opens up to space in a way that the viewer feels the immersion to a Mona Lisa 3D cube. The digital and spatial nature of Yvaral's Mona Lisa fostered discussion about possible implications in pre-school education.

The above examples of appropriating Mona Lisa show how this portrait has been the center of dialogue and discussion among artists and different artistic movements. Marcel Duchamp's saying "Art is a game among all people of all eras" (Bourriaud, 2002, p.18) comes true in the case of Mona Lisa which gave rise to a lot of discussions and inspired creativity to people of subsequent years and centuries.

\section{Contemporary Technology and Digital Mona Lisa}

Despite its being a historical cinquecento portrait, Mona Lisa goes hand-in-hand with contemporary technology -digital and otherwise- by appearing in numerous forms and parodies with messages indicative of up-to-date situations and tendencies. A black Mona Lisa, a bald Mona Lisa for the campaign against cancer, a sexy Mona Lisa, a Superman Mona Lisa, multicultural Mona Lisa (Pythagoreio Kindergarten, 2014), a Mona Lisa taking selfie with a smartphone, a Mona Lisa with headphones, Mona Lisa Facebook profile pictures with the person's face in the form of Mona Lisa are indicative examples rendering this portrait continuously popular and always contemporary.

Figure 1 is from a museum exhibition on Leonardo Da Vinci and shows the different versions of Mona Lisa which endow the portrait with an increasingly popular, intercultural and broader (i.e. further than digital) technological character. More precisely, in Figure 1 one comes up with a playmobil Mona Lisa, a LEGO Mona Lisa, a Mona Lisa Simpson, Mona Lisa as Virgin Mary, cubist Mona Lisa, Botero Mona Lisa etc. What came out from the discussion with students was that the playfulness as well as the characters of these parodies (Minions, playmobil, etc.) are expected to render Mona Lisa popular to pre-schoolers. 


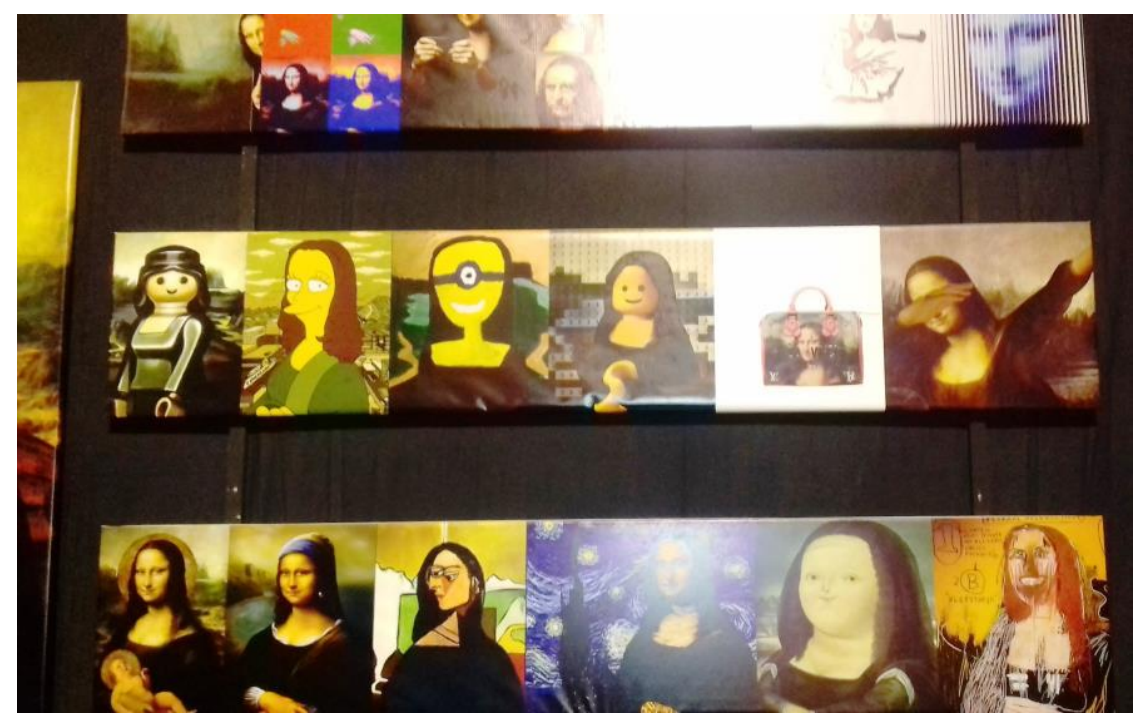

Figure 1: Mona Lisa reproductions and parodies, 2019, photo from the exhibition Leonardo Da Vinci: the Man, the Art, the Genius, Tsalapata Complex, Volos, Greece

\section{Collective Mona Lisa: the case of a distance education art-and-technology project}

\section{Project framework and objectives}

Collective Mona Lisa art-and-technology project was carried out in a higher education Department of Early Childhood Education in Greece. It was created during the COVID-19 pandemic in a distance education framework, i.e. in the synchronous digital classroom. The designing of objectives took into account the need for students to develop digital, participatory and collaborative skills in the distance education condition. It also took into account the students' prior knowledge in the field of the history of art. More precisely, since this activity was carried out with students from a Department of Education, the choice of Mona Lisa as a famous portrait that all students already knew was expected to make them feel safe, confident and free to work on this portrait and transform it with the use of digital tools.

The initial and basic objective for students was to learn how to use digital tools - mainly transform tools - in order to obtain skills in digital image processing. Another objective for students was to get familiar with Renaissance painting and also other art movements and art-and-technology tendencies in an enjoyable way through the history of the original Mona Lisa portrait followed by scientific tests and numerous examples of its reproductions, appropriations and parodies as well as through creating their own digital transformations. However, digital creativity was not limited to the individual level. Instead, another important objective of this activity was to move from self-expression to a collective art making experience and to promote dialogue with regards to art and technology perception and practice. The collectivity of this creative experience was of high importance especially during the COVID-19 pandemic circumstances of distance education and loss of physical contact between the Professor and the students as well as within the students' community. Further literature on distance education art-and-technology projects is expected to flourish during or after COVID-19 pandemics. 


\section{Project methodology}

The theoretical background of the Collective Mona Lisa art-and-technology project was based on: the constructivist learning theory, the experiential learning, the art-based learning, the transdisciplinarity in arts, the STEAM (Science, Technology, Engineering, Arts and Mathematics) approach and the appropriation (see Section 1) in art and visual culture. The case study of Collective Mona Lisa workshop is a qualitative research whose implied methodology involved: visual (formal) analysis of the artworks (see Section 1) and digital activities (see Section 3.3.). 22 students participated in the project.

The constructivist learning theory influenced and determined the selection of Mona Lisa for this project. The fact that all students already knew Da Vinci's Mona Lisa was crucial for their confidence in the discussions as well as in the creative process. Their daily use of digital technology also contributed to the increase of their confidence. Based on these two parameters, in Collective Mona Lisa the initial level of the students along with the link with their 'zone of proximal development' (Vygotsky, 1978, p. 86) were taken into account with the intention to gain confidence, willingness and motivation for expression through digital art.

According to Paulo Freire:

"the humanist, revolutionary educator's [...] efforts must be imbued with a profound trust in people and their creative power. To achieve this they must be partners of the students in their relations with them." (Freire, 1970, 1993, p.75)

Collective Mona Lisa was based on the creativity of its participants and the author/instructor was an active partner of the students. The author/instructor also actively participated in the creation of Collective Mona Lisa and there are digital images of hers next to the ones of the students in Figures 2 and 3. Experiential learning is active rather than passive and promotes active engagement in many ways (Beard, 2010). In Collective Mona Lisa active engagement was fostered through discussions about Mona Lisa original painting, (re)interpretations and parodies as well as through students' individual digital creations and collective digital montages.

The art-based learning is not only 'instrumental' in the sense that it is not only about the materials, processes and creativity in and through the arts. Instead, art-based learning also takes into account the overall art's contribution to learning and development through its connections with the context and environment of the creators. (Sullivan, 1993). The present paper takes deeply into account the context, i.e. the special circumstances of the COVID-19 pandemics which do not permit trips or museums visits. Thus, in a time when it is impossible to undertake a physical museum visit, especially in Louvre which would require a trip to Paris, a distance education project about Mona Lisa is put forward. Collective Mona Lisa connects with digital art, digital creative technologies and collective and participatory art-and-technology experience in the framework of distance education. Collective Mona Lisa project with the implication of many senses, intelligences, disciplines and learning styles in combination with a deep inquiry from the part of the students and active participation of both students and the author/instructor increase options, interest and motivation (Fleming, 2000, p.11).

The transdisciplinarity in arts is a sine qua non in Collective Mona Lisa project where image processing promotes students' visual and digital literacy and opens them up to mathematics (algebra and geometry) as well as to learning and deeper comprehension of Greek and English language 
through the names of the transform tools and effects of the software. However, transdisciplinarity lies not only on the digital nature of the project, which requires the use of technology, but also on the (re)use and appropriation of Mona Lisa and the consequent need to know the historical context of Da Vinci's portrait. Furthermore, it was essential to cover the subject of Mona Lisa's subsequent replicas, (re)interpretations and parodies in order to connect with the history of art and the contemporary condition. According to Vaos art is undivided with other areas of knowledge and practice and leads to a holistic approach towards reality, so art making and art perception need synergies of different disciplines and fields (Vaos, 2008, p.40-41).

STEAM approach came from adding the arts (A) to STEM. The addition of the "A" benefits students by enhancing their creative skills and contributes to a better and deeper understanding of STEM (Souliotou, 2016). Through arts students learn in an engaging and enjoyable way. It goes without saying that Collective Mona Lisa participatory digital art project involves art and technology, i.e. the 'A' and 'T' of the STEAM acronym. Collective Mona Lisa, as stated above, also links with the 'M', i.e. Mathematics, through the use of algebra and geometry in digital design. Furthermore, the presence of the 'S', i.e. Science, is also obvious through the scientific imagery of Mona Lisa which was showed and discussed with students.

\section{Project development}

At first students saw the picture of the original painting and imagery from its scientific analyses in Louvre's website (https://focus.louvre.fr/en/mona-lisa/compare/scientific-tests). They were also given examples of appropriations and parodies of Mona Lisa from the history of art as well as from the visual culture. The history of these appropriations and parodies is analytically presented in the above sections 1 and 2. An intensive discussion followed about: the fame of Mona Lisa; the adventure of seeing the original painting in the crowded Louvre and the possibility of seeing Mona Lisa through a virtual tour, which is the only possibility during pandemics; ways to learn and to notice every detail on https://focus.louvre.fr/en/mona-lisa. Furthermore, students were very much impressed by the numerous transformations and parodies of Mona Lisa both in the Modern and the Contemporary art scene as well as in the visual culture, scientific and commercial imagery. A particularly long discussion was made especially with regards to Composite Mona Lisa digital installation by JeanPierre Yvaral. Students stated that it is possible to include this installation in activities with preschoolers for the development of numeracy, visual-spatial and digital skills.

Then students gave their own creative 'responses' to all the above imagery which was inspired by the original Mona Lisa portrait. More precisely, students expressed themselves through making digitally transformed images, animated pictures (Graphic Interchange Format - GIFs) and short videos of Mona Lisa which they afterwards put together -with the supervision and guidance of the author/instructorin collective digital montages. Students used contemporary digital technology, design software and platforms which enable various transformations using filters, textures, effects and distortions of digital images. Clones, distortions, semi-transparencies, repositions and other transformations within 75 Mona Lisa versions (66 + 9 versions in Figures 2 and 3) render their collective artwork a digital art composition which shows these multiple possibilities and forms of digital design and transform tools.

Figure 2 is composed of 66 transformations where Mona Lisa appears with distorted face expressions, changed positions (e.g. the upside-down Mona Lisa, the horizontally flipped Mona Lisa, the vanished 
Mona Lisa, etc.), multiple faces or disproportionate face in relation to body parts. The repetition of the original rectangular format in Collective Mona Lisa: grid implies a reference to pixel structure of digital images. In Collective Mona Lisa: sequential perspectives (Figure 3) different perspectives of Mona Lisa are put together in a digital montage which explicitly opens up to the third dimension. It is important to note that this montage highly contributed to students' better comprehension of perspective and anamorphosis. Although both these phenomena are known from physical experience, they are transcribed into the digital form in Collective Mona Lisa: sequential perspectives. Furthermore, the manipulation of digital images when altering perspective enabled students to see Mona Lisa from multiple viewpoints and to choose the viewpoint which they found more characteristic, interesting or appealing.

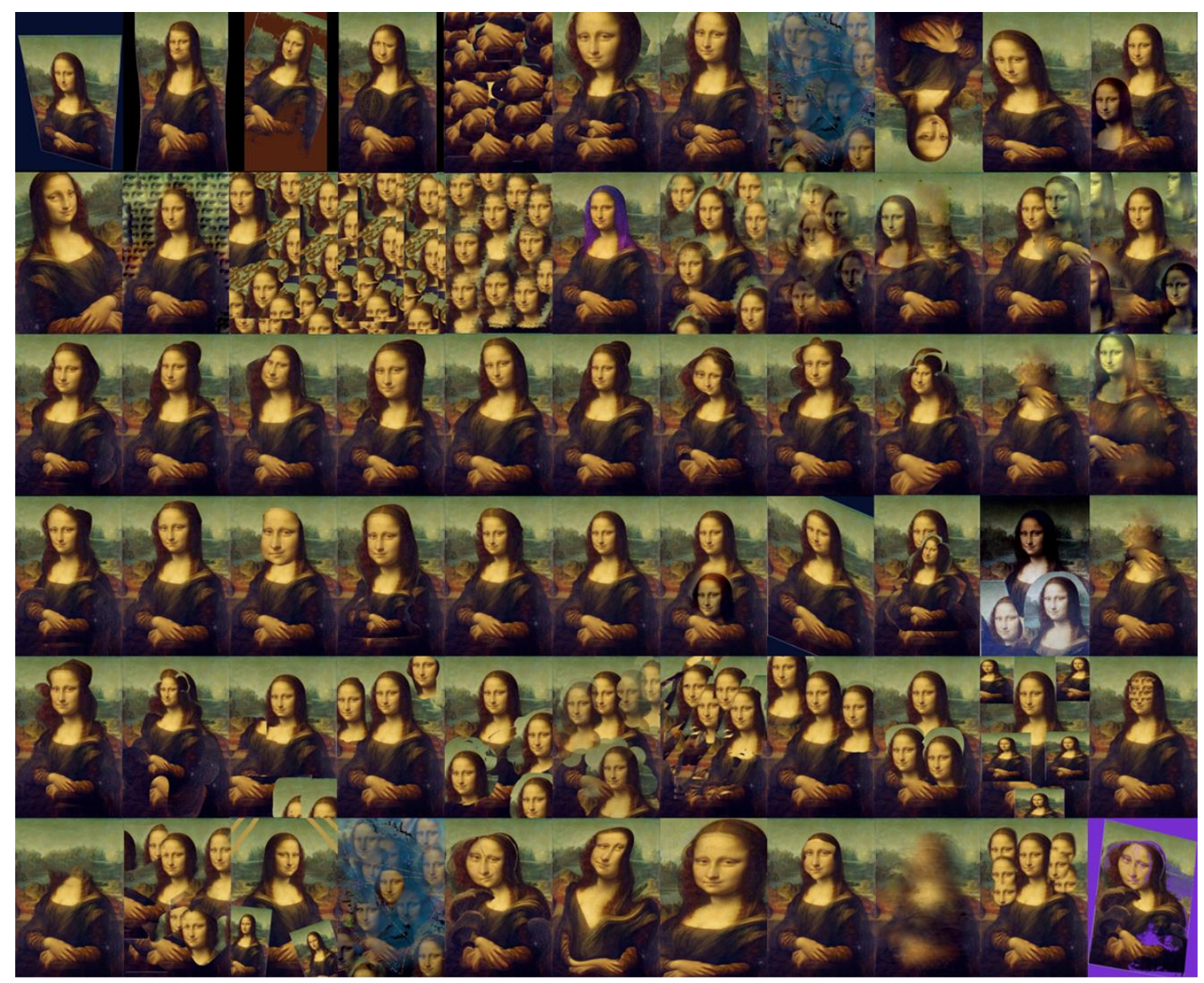

Figure 2: Collective Mona Lisa: grid, 2020, 66 digital transformations of Mona Lisa made by the author/instructor and 22 students. 


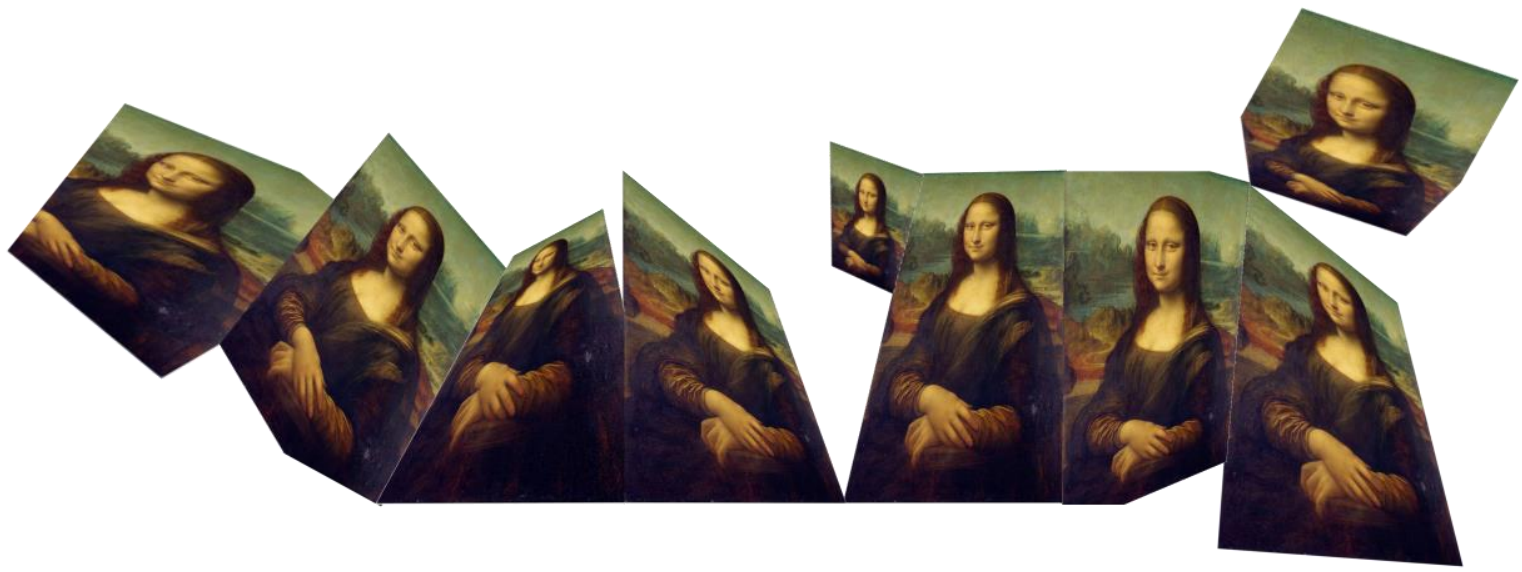

Figure 2: Collective Mona Lisa: Sequential Perspectives, 2020, 9 digital transformations of Mona Lisa made by the author/instructor and 5 students.

\section{Discussion and Conclusions}

Mona Lisa's unreachable popularity makes it difficult for viewers to think beyond its representations on postcards, other products and advertisements and to see this portrait "with fresh eyes" (Gombrich, 1950, p.218-219) However, in the case of Collective Mona Lisa students are not any more passive viewers of Mona Lisa nor mere consumers of its reproductions and parodies on products or advertisements. Instead, students critically reflect on the possibilities of image transformation in art, visual culture, science and creative technology. Then, they go through their own transformations and image processing of Mona Lisa which they put together in individual and collective digital artworks. Thus, by appropriating a well-known High Renaissance painting masterpiece they become more familiar with the art tradition and at the same time they also become active learners and (co)creators of individual and collective artworks in the form of digital images and videos.

Students' collective artwork contributed to the deeper understanding of Da Vinci's masterpiece and increased their confidence and familiarity with Renaissance painting. The case of this activity proves that digital culture is a catalyst for art history learning and creativity in the virtual classroom. Furthermore, this activity fosters collaborative learning through distance education and turns out to be a vehicle for empowering learners in a digital world, as well as for developing linguistic, numerical and multisensory skills through digital creativity. COVID-19 pandemic urges the development of distance education collaborative methods and creative projects like Collective Mona Lisa in order to maintain students' interest and achieve community interaction and resilience. Thus, it is expected that many participatory distance education digital art projects and educational activities will be undertaken in near future. This kind of projects could take various forms and in some cases they could be inspired by famous Renaissance artworks which, in turn, would be proved as catalysts for creativity and deeper thinking.

Collective Mona Lisa project (re)uses and digitally transforms Mona Lisa (Figures 2 and 3). The (re)use of images with the transformation of the traditional original is inscribed in appropriation/adaptation (TATE, 2020) art practice which radically places tradition in the contemporary condition (Verwoert, 2007). There are many contemporary artists who embrace this kind of art practice by creating digital or other contemporary artistic responses to famous artworks of the past. Focussing on artists with appropriation/adaptation responses to Renaissance artworks, it is 
worth mentioning, among many others, Andy Warholl's Venus after Botticelli (1984), Hans-Peter Feldmann's David (2006) which is an appropriation/adaptation of Michelangelo's homonymous statue, Jeff Koons' Gazing ball (da Vinci Mona Lisa) (2015) and his series of gazing ball paintings (Koons, 2021).

Another important aspect of Collective Mona Lisa project is that it brings together the traditional with the digital. Although the traditional is often opposed to the digital and the modern, this project indicates that it is possible and interesting to achieve harmonic coexistence of the traditional and the digital. (Weiming et al, 2010). This leads to a modern, contemporary and up-to-date view of the traditional as well as to a (re)consideration of the digital through tradition. Thus, the present paper proves that one can definitely see Mona Lisa "with fresh eyes" through creativity. Collective Mona Lisa is a digitally traditional and traditionally contemporary project which renders art-based education and distance-education cutting-edge forms for collaborating and learning.

It is interesting to note that Da Vinci's $2 u v r e$ and personality epitomized the efforts of artists to prove that painting is equal with liberal arts (Lampraki-Plaka, 2021). 500 years later Collective Mona Lisa and many other contemporary art-and-technology projects are examples which prove that visual arts go hand-in-hand with contemporary technology, science and other fields of human knowledge and thought with the possibility of creatively empowering learners and render them versatile in the contemporary world. In Collective Mona Lisa activity learners are future educators of children, so their empowerment in the digital world should set the ground for inspiring children's creativity through art-response and collaborative learning through digital co-creation and active participation.

Collective Mona Lisa also actively connects visual arts with STEM by using computer graphics and computational geometry. This combination of art, creative technologies and mathematics is at the heart of creative industries and STEAM approach in education. (Kalovrektis et al, 2021, p.55) Collective Mona Lisa also proves that digital technologies lead to the development of linguistic (e.g., the vocabulary of the digital tools, transform tools and effects), numerical (e.g., metrics, measurements etc.) and multisensory skills (e.g., visual transformations and effects, audio and audio effects in videos) through digital creativity.

Furthermore Collective Mona Lisa fosters community resilience in times of COVID-19 pandemics. Students see their individual work as well as the work of their colleagues in a common collective artwork and the sense of community is, thus, empowered.

\section{References}

Augustyn, A. (2021). The High Renaissance. In Britannica, Retrieved February, 7, 2021, from https://www.britannica.com/event/Renaissance/The-High-Renaissance

Belton, R.J. (1996). Art History: a Preliminary Handbook. Why Study Visual Culture? The University of British Columbia - Okanagan Campus. Retrieved from https://fccs.ok.ubc.ca/studentresources/arth/

Bourriaud, N. (2002). Postproduction - Culture as screenplay: how art reprograms the world. New York: Lukas \& Sternberg.

Cadet, C. (1887). Le Rire. Le Rire, $2^{\text {nd }}$ ed., 5. 
Centre Pompidou, Direction de l'action éducative et des publics. (2005) L'œuvre de Marcel Duchamp. Retrieved February, 7, 2021, from http://mediation.centrepompidou.fr/education/ressources/ENSDuchamp/ENS-duchamp.htm

Cole, M., John-Steiner, V., Scribner, S. \& Souberman, E. (1978). L. S. Vygotsky. Mind in society: the development of higher psychological processes. Cambridge, Massachusetts and London, England: Harvard University Press, 86.

Craven, D. (2017). Marcel Duchamp and the perceptual dimension of conceptual art. In B. Winkenweder (Ed.) Art history as social praxis: the collected writings of David Craven. Boston: Brill, 75.

Fleming, D. (2000). A teacher's guide to project-based learning. Charleston, West Virginia: AEL, 11.

Freire, P. (1993). Pedagogy of the oppressed. $30^{\text {th }}$ anniversary edition. London, New York: The Continuum International Publishing Group Inc., 75.

Freire, P. (1970). Pedagogy of the oppressed. London, New York: The Continuum International Publishing Group Inc., 75.

Gombrich, E.H. (1950). The story of art. New York, NY: Phaidon Publishers Inc.

Kalovrektis, K., Xenakis, A., Psycharis, S., Stamoulis, G. (2021). Educational Technology, Developing platforms of robotics and IoT. Thessaloniki: Tziolas Publications, 55. (in Greek)

Koons, J. (2021, April 23). Jeff Koons: Gazing Ball Paintings (da Vinci Mona Lisa). Retrieved from http://www.jeffkoons.com/artwork/gazing-ball-paintings/gazing-ball-da-vinci-mona-lisa

LaFarge, A. (1996). The Bearded Lady and the Shaven Man: Mona Lisa, Meet" Mona/Leo". Leonardo, 29(5), 379-383. doi:10.2307/1576403

Lampraki-Plaka, M. (2021, February 4). Faces of the Hero: Uomini Famosi in Italian Renaissance. Humanists and Artists. Stavros Niarchos Foundation Cultural Center, Athens, Greece. Retrieved from https://www.youtube.com/watch?v=qnIFFoOpQBg

Le fumisme. (2021). Retrieved from https://www.lemonde.fr/blog/correcteurs/files/2015/03/DSCN1309.jpg (in French)

Musée de Montmartre. (2021). Retrieved from https://museedemontmartre.fr/ (in French)

Picabia, F. (1920, March). Tableau DADA par Marcel Duchamp L.H.O.O.Q. Manifeste DADA. 391. (in French)

Popper, F. (1993). Art of the electronic age. New York: Harry N. Abrams

Pythagoreio Kindergarten, (2014, October 5). Mona Lisa is not only from Italy! Retrieved from http://pythagoreionip.blogspot.com/search?updated-min=2014-01-01T00:00:00-08:00\&updated$\underline{\max =2014-10-06 \mathrm{~T} 04: 01: 00-07: 00 \& \max -\text { results }=44 \& \text { start }=6 \& \text { by-date }=\text { false }}$

Ritchhart, R., Church, M., Morrison, K. (2011). Making thinking visible: how to promote engagement, understanding and independence for all learners. San Francisco, CA: Jossey-Bass, A Wiley Imprint.

Sayre, H. M. (2006). Writing about Art. Upper Saddle River, NJ: Pearson/Prentice Hall.

Scailliérez, C. (2021, February 7). Mona Lisa - Portrait of Lisa Gherardini, wife of Francesco del Giocondo. Retrieved from https://www.louvre.fr/en/oeuvre-notices/mona-lisa-portrait-lisa-gherardiniwife-francesco-del-giocondo

Souliotou, A. Z. (2016). The importance of including the Arts in STEM education, Hellenic Conference on Innovating STEM Education [HiSTEM 2016], National and Kapodistrian University of Athens. Retrieved from https://stemeducation.upatras.gr/histem2016/ (in Greek)

Steiner, W. (1987). Postmodernist Portraits. Art Journal, 46(3), 173-177. doi:10.2307/777029

Sullivan, G. (1993). Art-Based Art Education: Learning That Is Meaningful, Authentic, Critical and Pluralist. Studies in Art Education, 35(1), 5-21. doi:10.2307/1320834, p.16, 19. 
TATE. (2021). Art term: Appropriation. In TATE online glossary. Retrieved February, 12, 2021, from https://www.tate.org.uk/art/art-terms/a/appropriation

Vaos, A. (2008). Issues of Didactics of Visual Arts: the art venture as pedagogical praxis. Athens: Topos. (in Greek)

Verwoert, J. (2007) 'Apropos Appropriation: Why Stealing Images Today Feels Different', Art \& Design Research A Journal of Ideas, Context and Methods, 1. (2), pp.1, 2/05/2013, Retrieved from http://www.artandresearch.org.uk/v1n2/verwoert.html

Weiming, D., Zhongyang, L., Qian G., (2010). "Analysis of the interaction between digital art and traditional art," 2010 International Conference on Networking and Digital Society, Wenzhou, 2010, pp. 441-443.

Wilhelm, J.D., Douglas, W., Fry, S. W. (2014). The Activist Learner: Inquiry, Literacy, and Service to make learning matter. New York, NY: Teachers College Press, Columbia University 